\title{
Preface
}

\section{Jaume Llibre}

Published online: 27 February 2010

(C) Birkhäuser / Springer Basel AG 2010

This special issue of Qualitative Theory of Dynamical Systems is an outgrowth of the fifth international symposium on Hamiltonian Systems and Celestial Mechanics (HAMSYS-2008) which was held in Guanajuato, México, July 7-11, 2008. The scientific organizers were, in alphabetical order, J. Delgado, E. A. Lacomba, J. Llibre and E. Pérez-Chavela. The third one from Universitat Autónoma de Barcelona and the others from Universidad Autónoma Metropolitana-Iztapalapa. This was the fifth of a series of similar symposia whose goal is to gather top researchers in the area of Celestial Mechanics and Hamiltonian Systems.

As in the previous ones the main idea was to communicate new results and look forward for future lines of research. For graduate students in the field, this conference offered the opportunity of personal contact with the experts to help them in their own research.

The papers in this volume include topics such as central configurations, relative equilibria and their stability, dynamics and numerics of few body problems in Newtonian and other potentials, regularization, non-integrability and also some papers related to aspects of quantum mechanics and complex dynamical systems.

During the symposium there was a special session of open problems to motivate joint research in the above topics and also as possible themes of doctoral theses for young students. There were about 40 senior researchers and 20 graduate students from several countries including, USA, France, Spain, Japan, Canada, Brazil and Colombia.

All papers published in this special issue of QTDS went through a strict refereeing process according to the editorial standards of the journal.

We thank CONACYT-México, grant 47768; DGICYT-Spain, grant; Sociedad Matemática Mexicana, Departamento de Matemáticas UAM-Iztapalapa. Special

\footnotetext{
J. Llibre $(\varangle)$

Barcelona, Spain

e-mail: jllibre@mat.uab.cat
} 
thanks are due to the director of CIMAT, José Carlos Gómez Larrañaga and to Renato Iturriaga for both their hospitality and help in the local logistics of the Symposium. We also appreciate the collaboration of José Castro for his administrative help during the event. Al last but not least, we deeply thank all the participants of the symposium for the amazing, intense and highly productive week of mathematical experience.

J. Delgado, E. A. Lacomba, J. Llibre, E. Perez-Chavela Guest Editors 